\title{
Dosimetric study of SIB-IMRT versus SIB-3DCRT for breast cancer with breath-hold gated technique
}

\author{
Suresh Moorthy ${ }^{1}$, Hamdi Sakr ${ }^{1}$, Shubber Hasan ${ }^{1}$, Jacob Samuel ${ }^{1}$, Shaima Al-Janahi ${ }^{1}$, \\ Narayana Murthy ${ }^{2}$ \\ ${ }^{I}$ Department of Oncology \& Hematology, Salmaniya Medical Complex, Kingdom of Bahrain \\ ${ }^{2}$ Department of Physics, Acharya Nagarjuna University, Guntur, India
}

Received September 05, 2013; Revised October 02, 2013; Accepted October 08, 2013; Published Online October 10, 2013

\section{Original Article}

\begin{abstract}
Background and purpose: 3-dimensional conformal therapy (3DCRT) is widely employed radiation therapy technique for breast cancer, but there is still need to minimize the doses to organ at risk (OAR) using 3DCRT. A few clinical studies have discussed using intensity modulated radiation therapy (IMRT) to address this shortfall. Simultaneous integrated boost (SIB) has been used in head and neck and prostate cancer, and there is a growing interest in using SIB for breast cancer too. This study aimed to compare SIB-IMRT versus SIB-3DCRT for breast cancer patients. Materials and Methods: SIB-3DCRT treatment plans were created for 36 consecutive patients. Dose was prescribed as $45 \mathrm{~Gy}$ in 25 fractions to the planning target volume (PTV)-1 and 60 Gy in 25 fractions to PTV-2. Treatment plans were normalized to 95\% of PTV volume receiving 95\% of the prescription dose. The conformity index (CI), homogeneity index (HI), lung dose, heart dose, left anterior descending artery(LAD) dose, and low dose volume and integral dose of normal healthy tissue were recorded and analyzed. Results: With the use of IMRT technique, there was an improvement in CI (0.14) when compared to CI of 3DCRT $(0.18 ; \mathrm{p}=0.01)$. However, there was no significant difference in the HI ( $\mathrm{p}=0.45)$. On average, the V20Gy of ipsilateral lung was $37.9 \%$ for 3DCRT and $22.4 \%(\mathrm{p}<0.01)$ for IMRT, whereas the $\mathrm{V}_{20 \mathrm{~Gy}}$ of total lung (ipsilateral + contralateral) was $21.8 \%$ for 3DCRT and $12.14(\mathrm{p}<0.01)$ for IMRT. Similarly, average $\mathrm{V}_{40 \mathrm{~Gy}}$ of heart was $7.5 \%$ for 3DCRT and $2.13 \%(\mathrm{p}=0.01)$ for IMRT. The LAD maximum dose to left side breast patients, on average, was $39.5 \mathrm{~Gy}$ for 3DCRT and $29.17 \mathrm{~Gy}(\mathrm{p}=0.03)$ for IMRT. The average number of monitor units was about 180 for 3DCRT and $1441(\mathrm{p}<0.01)$ for IMRT. Conclusion: IMRT for breast cancer treatment is feasible. In comparison to 3DCRT, IMRT can reduce the maximum dose to the target volume, and dose to the OAR. However, 3DCRT technique is superior in terms of low dose volume, integral dose, and treatment time. With the use of breath-hold gated technique in IMRT, it can further improve the target coverage and reduction of doses to the heart, lung, and LAD. SIB technique could reduce the overall treatment duration by about one week.
\end{abstract}

Keywords: Intensity Modulated Radiation Therapy, Three Dimensional Conformal Radiotherapy, Simultaneous Integrated Boost, Breath-Hold, Technique, Breast Cancer

\section{Introduction}

Breast cancer is the most common malignancy in women. Radiotherapy is an integral part of breast cancer management either in breast conservation surgery (BCS) or in post

Corresponding author: Suresh Moorthy, M.Sc, M.Phil; Division of Radiation Oncology, Department of Oncology \& Hematology, Salmaniya Medical Complex, MOH, Kingdom of Bahrain. Email: nmsureshm@gmail.com

Cite this article as: Moorthy S, Sakr H, Hasan S, Samuel J, Al-Janahi S, Murthy N. Dosimetric study of SIB-IMRT versus SIB-3DCRT for breast cancer with breath-hold gated technique. Int J Cancer Ther Oncol 2013;1(1):010110. DOI: $10.14319 /$ ijcto.0101.10 mastectomy cases. Many prospective studies have shown that adjuvant radiotherapy improves local control and survival rate in breast cancer patients after surgery. ${ }^{1}$ During earlier days of radiotherapy, opposed wedged fields with half beam block was considered as the standard radiation therapy technique. In the last decade, an introduction of linear accelerators has made 3-dimensional conformal radiotherapy (3DCRT) as a standard treatment technique, which can re-duce the doses to the lung, heart, and other critical structure doses in the breast cancer treatment. However, using 3DCRT, it is not always possible to achieve adequate normal tissue sparing, especially when treating left side chest wall patients. This is mainly due to overlying concave shape of 
the target, which can result more doses to adjacent structures such as heart and lung. Hong et al. ${ }^{2}$ compared intensity modulated radiation therapy (IMRT) with $3 \mathrm{D}$ conformal tangential wedged beams, and showed the reduction of dose to the coronary arteries, contra lateral breast, ipsilateral lung, and surrounding soft tissues using IMRT. By modulating photon beam, it is possible to obtain concave and convex shape dose distributions with IMRT, and it has the ability to conform radiation dose to irregular target volumes sparing the underlying critical structures resulting in better tumor control probability (TCP) and reduced normal tissue complication probability (NTCP). The main purpose of this study was to further evaluate normal tissue sparing and dosimetric analysis of simultaneous integrated boost (SIB)-3DCRT and SIB-IMRT in breast patients, with focus on breath-hold gated technique.

\section{Materials and Methods}

In this retrospective treatment planning study, we used computed tomography (CT) data of 36 consecutive patients with breast cancer post lumpectomy (18 left sides and 18 right sides), and all patients were treated with respiratory gated technique for breast radiotherapy.

\section{CT Simulation}

All 36 patients were simulated using 4D CT scanner (Philips Medical Systems, Andover, MA, USA) with whole-body Vaclok (Civco Medical Solutions, Iowa, USA) immobilization system. Patients were positioned on a wide bore CT-SIM couch with the help of lasers, and both arms of the patient were raised above patient's head. Furthermore, radio opaque markers were placed during the immobilization procedure to guide the isocenter shift. For all the patients, CT scans images were obtained from mandible to upper abdomen area with intravenous contrast, and CT scans were obtained using slice thickness of $5 \mathrm{~mm}$. Prior to CT simulation, patients were given training on breath-hold technique.

\section{Target Delineation and Dose Prescription}

After the CT simulation, the Digital Imaging and Communications in Medicine (DICOM) images were transferred to Eclipse treatment planning system (TPS) (version 10.0.34, Varian Medical Systems, Palo Alto, California, USA). Clinical target volume (CTV), planning target volume (PTV) and Organ at Risk (OAR) volumes were delineated on the axial CT slices. The lumpectomy gross tumor volume (L-GTV) was contoured using all available clinical and radiographic information including the excision cavity volume, architectural distortion, lumpectomy scar, seroma and/or extent of surgical clips.

CTV1 included the palpable breast tissue demarcated with radio opaque markers at CT simulation. The apparent CT glandular breast tissue visualized by CT, consensus definitions of anatomical borders, and the lumpectomy CTV from the RTOG breast cancer atlas. The breast CTV is limited anteriorly within $3 \mathrm{~mm}$ from the skin and posteriorly to the anterior surface of the pectoralis, serratous anterior muscle excluding chest wall. PTV was created by 3D expansion of CTV1 by $7 \mathrm{~mm}$. CTV2 was created by $1 \mathrm{~cm}$ 3D expansion from L-GTV and was limited posteriorly at anterior surface of the pectoralis and antero-laterally $3 \mathrm{~mm}$ from skin. PTV2 was created by $7 \mathrm{~mm} \mathrm{3D}$ expansion of CTV2. The normal structures were contoured as ipsilateral lung, contra lateral lung, contra lateral breast, heart, left anterior descending (LAD) artery, spinal cord, esophagus, trachea, humerus head, and liver. Dose prescription was applied per International Commission on Radiological Units and Measurements (ICRU) 50 and 62.3.4 Specifically, dose was prescribed as 45 Gy in 25 fractions $(1.8 \mathrm{~Gy} /$ fraction) to the PTV-1 and $60 \mathrm{~Gy}$ in 25 fractions (2.4Gy/fraction) to PTV-2.

\section{Treatment planning}

For treatment planning, 6 mega-voltage (MV) X-rays from Clinac 600CD linear accelerator (Varian Medical Systems, Palo Alto, California, USA) integrated with 120 leaves millennium multi-leaf collimator (MLC) was used. For the dynamic IMRT plans, 7 non-coplanar beams were used to achieve the minimum criteria of $95 \%$ of the volume received $95 \%$ of the prescribed dose. The treatment fields were almost evenly spaced within an arc of $180^{\circ}$ on the side of the tumor. Gantry angles ranged from $330^{\circ}$ to $150^{\circ}$ (clockwise) for the left side tumors and from $50^{\circ}$ to $210^{\circ}$ (counterclockwise) for the right side tumors. In Eclipse TPS, the IMRT plans were created with inverse plan optimization, and the algorithm used was Dose Volume Optimizer (version 10.0.28). For the dose calculation, pencil beam convolution (PBC) algorithm (version 10.0.28) was used, and leaf motions were calculated with leaf motion calculator (LMC) algorithm (version 10.0.28). Heterogeneity correction was done using modified Batho method in the Eclipse. For plan optimization, OAR dose constraints were given as ipsilateral lung $\mathrm{V}_{20}<30 \%$, heart $\mathrm{V}_{30}, \mathrm{~V}_{40}$, and mean dose as low as possible, contra-lateral breast mean dose less than $5 \mathrm{~Gy}$, and spinal cord maximum point dose less than $40 \mathrm{~Gy}$. For the 3DCRT plans, 4 to 6 non-coplanar beams were used to produce adequate dose coverage for the PTV. Critical organs were shielded using MLC without compromising PTV coverage. Beam weights were adjusted until the optimum coverage and acceptable hot spots were achieved. Additionally, field-in-field was created to reduce hotspot equal to or lower than $110 \%$ as well as to improve the target coverage and homogenous dose distribution in the PTV.

\section{Plan evaluation}

Dose-Volume Histograms (DVH) was used to analyze the volume receiving $20 \mathrm{~Gy}, 30 \mathrm{~Gy}$ and $40 \mathrm{~Gy}$, mean, maximum and minimum doses. The target dose uniformity and conformity were calculated and evaluated. Different scoring indices were given by various authors. ${ }^{5-7}$ In this study, we have followed indices defined by ICRU $83 .{ }^{8}$ 
The conformity index (CI) as defined in ICRU 83 is

$\mathrm{CI}_{\text {ref }}=\frac{\text { Volume of PTV covered by the reference dose }}{\text { Volume of PTV }} \quad$ Eq. 1

$\mathrm{CI}=1.0$ is ideal value

The Homogeneity Index (HI) as defined in ICRU 83 is

$\mathrm{HI}=\frac{\mathrm{D}_{2 \%}-\mathrm{D}_{98 \%}}{\mathrm{D}_{50 \%}} \quad$ Eq. 2

Where, D 2\%, D 98\%, D 50\% is dose received by $2 \%$, $98 \%, 50 \%$ volume. $\mathrm{HI}=0$ (Zero) is ideal value. Also, to illustrate the low dose volume effect, $V_{5 G y}$ volume and integral dose were calculated for normal healthy tissue.

Integral Dose $=$ Mean Dose $(\mathrm{Gy}) \times$ Volume $\left(\mathrm{Cm}^{3}\right) \quad$ Eq. 3

\section{Statistical Analysis}

Statistical Analysis was performed using the Wilcoxon Signed Rank test. This matched pair $t$ test was applied to determine the statistical difference between the dose-volume data for IMRT versus 3DCRT. The values are reported in ranges. The reported $\mathrm{p}$ value is two tailed, and $\mathrm{p}$ values of $<0.05$ are considered statistically significant.

\section{Results and Discussion}

Dose volume histograms of the normal tissues of both the plans (IMRT and 3DCRT) are presented in Table 1. The normalized target coverage of both treatment methods is presented in Table 2 and Table 4. The PTV mean dose for 3DCRT is 47.10 Gy compared to $45.88 \mathrm{~Gy}$ ( $\mathrm{p}<0.01$ ) with IMRT. The dose distribution in axial sections is shown in Figures 1 and 2. These axial sections clearly show that concave PTV coverage and exclusion of LAD during optimiza-

TABLE 1: Comparison of normal tissue dose volume parameters for Respiratory Gated IMRT and 3DCRT breast cancer patients (Statistics based on Wilcoxon Signed Rank Test). The values are averaged over 36 analyzed patients.

\begin{tabular}{|c|c|c|c|}
\hline Organ & Parameter & SIB-3DCRT & SIB-IMRT \\
\hline \multirow{3}{*}{ Ipsilateral lung } & V20Gy (\%) & 37.9 & 22.4 \\
\hline & V30Gy $(\%)$ & 32.24 & 16.08 \\
\hline & Mean (Gy) & 20.29 & 16.51 \\
\hline Heart & $\mathrm{V}_{40 \mathrm{~Gy}}(\%)$ & 7.5 & 2.13 \\
\hline Both Lung & $\mathrm{V}_{20 \mathrm{~Gy}}(\%)$ & 21.8 & 12.14 \\
\hline LAD & Max. Dose (Gy) & 39.5 & 29.17 \\
\hline
\end{tabular}

tion by IMRT. Also, previous studies have reported lower doses to the ipsilateral lung, contra lateral lung, contra lateral breast, heart, and LAD doses using IMRT technique. ${ }^{10,11}$

3D conformal plans using asymmetric jaw and field-in-field technique provides better coverage than a conventional physical wedged -half beam blocked or physical wedged-asymmetric fields. Furthermore, physical wedge has limitation in field width and lengths. With 3DCRT, the hot spots occurred in superficial skin surface, but IMRT exhibited better control in shifting the hot spots, with a possibility of keeping dose to the skin equal to or less than the prescription dose.

\section{Dose homogeneity and conformity}

The use of equally spaced gantry angles improved homogeneity and conformity indices as well as reduced the volume of critical normal tissues such as the heart and ipsilateral lung receiving a high dose as shown by Hong et al. ${ }^{2}$. In this study, we used equally spaced beam angles for both the IMRT and 3DCRT plans, and the average target maximum dose was lower with IMRT; however, it was not statistically significant. Although the mean breast volume in our study was $1221 \mathrm{cc}$, which is relatively higher compared to the literature ${ }^{12}$, we were able to demonstrate optimized coverage and reduced dose to the critical organs.

The inverse-planning IMRT further reduced hotspots mainly due to beam modulation during optimization compared to 3DCRT, where beam modulation is not available. Previous planning studies ${ }^{13,14}$ with multiple fields showed the PTV-95\% coverage values ranging from $90 \%$ to $97 \%$, whereas all our optimized plans had the PTV-95\% coverage values of $>95 \%$ of prescription dose. With the use of IMRT technique, our data showed that there is a consistent improvement in conformity index from 0.18 for 3DCRT to 0.15 for IMRT $(\mathrm{p}=0.01)$. However, there was no significant difference $(p=0.45)$ when HI of 3DCRT was compared to that of IMRT.

TABLE 2: Comparison of planning target volume (PTV1) coverage parameter for Respiratory Gated IMRT and 3DCRT breast cancer patients (Statistics based on Wilcoxon Signed Rank Test). The values are averaged over 36 analyzed patients.

\begin{tabular}{cccc}
\hline \hline PTV 1 Parameter & SIB-3DCRT & SIB-IMRT & $\mathrm{p}$ \\
\hline Minimum Dose (Gy) & 24.07 & 32.03 & $<0.01$ \\
Maximum Dose (Gy) & 51.97 & 59.68 & $<0.01$ \\
Coverage (\%) & 96.8 & 98.22 & $<0.01$ \\
Conformity Index & 0.18 & 0.14 & 0.01 \\
Homogeneity Index & 1.03 & 1.01 & 0.45 \\
Mean Dose (Gy) & 47.1 & 45.88 & $<0.01$ \\
Mod Dose (Gy) & 49.12 & 48.92 & 0.32 \\
Median Dose (Gy) & 50.5 & 48.8 & $<0.01$ \\
Stnd. Deviation(Gy) & 6.35 & 4.17 & $<0.01$ \\
V50Gy (\%) & 48.69 & 29.83 & $<0.01$ \\
V55Gy (\%) & 30.7 & 11.29 & $<0.01$ \\
\hline \hline
\end{tabular}




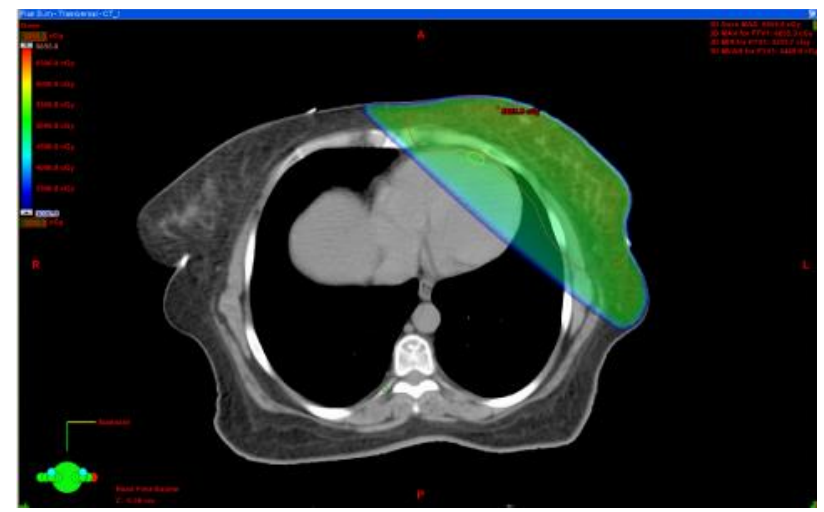

FIG. 1: Axial slice showing dose distribution in 3DCRT plan. The 3DCRT was unable to exclude LAD while covering the concave target, and increased dose to the heart and lung was noticed.

TABLE 3: Comparison of MU, ID and V5 parameter for Respiratory Gated IMRT and 3DCRT breast cancer patients (Statistics based on Wilcoxon Signed Rank Test). The values are averaged over 36 analyzed patients.

\begin{tabular}{cccc}
\hline \hline Parameter & SIB-3DCRT & SIB-IMRT & $\mathrm{p}$ \\
\hline Monitor Units & 180 & 1441 & $<0.01$ \\
Integral Dose $\left(\mathrm{Gy}-\mathrm{Cm}^{3}\right)$ & 145210 & 197428 & $<0.01$ \\
V5Gy $(\%)$ & 18.89 & 30.61 & $<0.01$ \\
\hline \hline
\end{tabular}

TABLE 4: Comparison of planning target volume (PTV2) coverage parameter for Respiratory Gated IMRT and 3DCRT breast cancer patients (Statistics based on Wilcoxon Signed Rank Test). The values are averaged over 36 analyzed patients.

\begin{tabular}{cccc}
\hline \hline PTV 2 Parameter & SIB-3DCRT & SIB-IMRT & $\mathrm{p}$ \\
\hline Minimum Dose (Gy) & 56.41 & 53.9 & $<0.01$ \\
Maximum Dose (Gy) & 64.9 & 64.01 & $<0.01$ \\
Coverage (\%) & 98.3 & 99.77 & 0.13 \\
Conformity Index & 0.12 & 0.08 & 0.01 \\
Homogeneity Index & 1.02 & 1.01 & 0.11 \\
Mean Dose (Gy) & 61.1 & 61.72 & 0.13 \\
Mod Dose (Gy) & 61.17 & 62.13 & $<0.01$ \\
Median Dose (Gy) & 61.99 & 62.45 & $<0.01$ \\
Stnd. Deviation(Gy) & 1.5 & 1.21 & 0.01 \\
\hline \hline
\end{tabular}

TABLE 5: Comparison of Non-Gated IMRT with respiratory Gated IMRT (mean) breast cancer patients (Statistics based on Wilcoxon Signed Rank Test). The values are averaged over 36 analyzed patients.

\begin{tabular}{cccc}
\hline \hline Parameters & $\begin{array}{c}\text { Non-Gated } \\
\text { SIB-IMRT }\end{array}$ & $\begin{array}{c}\text { Gated } \\
\text { SIB-IMRT }\end{array}$ & $\mathrm{p}$ \\
\hline LAD-Maximum Dose & $35.62 \mathrm{~Gy}$ & $29.17 \mathrm{~Gy}$ & $<0.01$ \\
Heart -V30Gy & $9.27 \%$ & $5.91 \%$ & $<0.01$ \\
Ipsilateral Lung-V20Gy & $30.2 \%$ & $22.4 \%$ & 0.03 \\
PTV -95\% of prescription & $96.81 \%$ & $98.22 \%$ & $<0.01$ \\
\hline \hline
\end{tabular}

In patients with breast cancer, it is intended that the irradiated heart volume be minimized to the greatest possible degree without compromising the target coverage. The risk of pericardial events is probably related to both dose and vol-

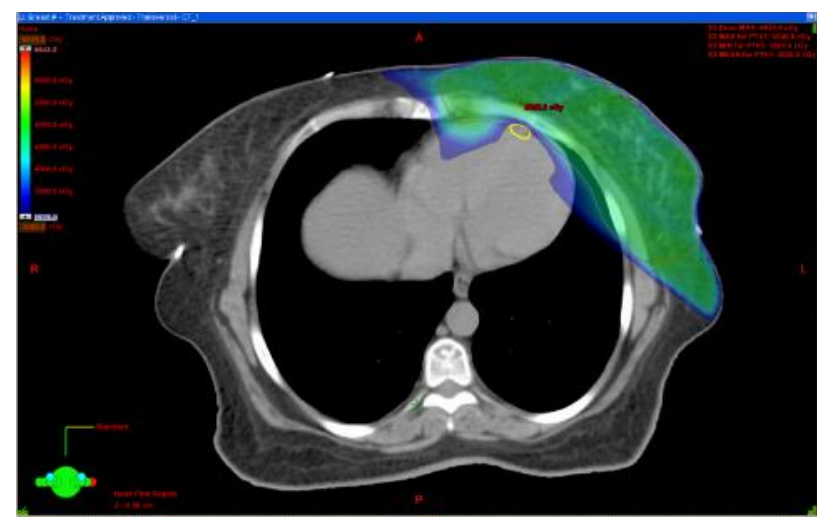

FIG. 2: Axial slice showing dose distribution in IMRT plan. The IMRT was able to exclude LAD while covering the concave target, and decreased dose to the heart and lung was noticed.

ume of radiation. The incidences of pericardial disease decrease with the use of sub cranial blocking the major ventricles at 30 Gy. Stewart et al. ${ }^{15}$ concluded that the dose should be limited to $60 \mathrm{~Gy}$ for less than $25 \%$ of cardiac volume and $45 \mathrm{~Gy}$ for more than $65 \%$ of cardiac volume.

In patients with breast cancer, it is intended that the irradiated heart volume be minimized to the greatest possible degree without compromising the target coverage. The risk of pericardial events is probably related to both dose and volume of radiation. The incidences of pericardial disease decrease with the use of sub cranial blocking the major ventricles at 30 Gy. Stewart et al. ${ }^{15}$ concluded that the dose should be limited to $60 \mathrm{~Gy}$ for less than $25 \%$ of cardiac volume and $45 \mathrm{~Gy}$ for more than $65 \%$ of cardiac volume.

In our study the heart $\mathrm{V}_{40 \mathrm{~Gy}}$ was significantly lower in IMRT than in 3DCRT $(\mathrm{p}<0.01)$, especially for left sided breast cancer patients, with mean heart $\mathrm{V}_{40}$ Gy of $7.5 \%$ for 3DCRT versus IMRT as $2.13 \%(\mathrm{p}=0.01)$. Gagliardi et al. ${ }^{16}$ reported that CAD risk was much reduced at doses less than $30 \mathrm{~Gy}$. Mean values of $\mathrm{V}_{30 \mathrm{~Gy}}$ were $<5 \%$ for IMRT compared with studies $^{17}$ reporting $\mathrm{V}_{30 \mathrm{~Gy}}$ values in the range of $2 \%$ to $5 \%$. Increased cardiac mortality risk associated with left side breast patients in the long term was reported by multiple authors. ${ }^{16}$, 18, 19 The advancement in treatment techniques such as IMRT has enabled to reduce cardiac exposure, and steady decline of radiation risk is being noticed. ${ }^{20}$ Furthermore, Boivin et al. ${ }^{21}$ noted that the anteriorly placed coronary arteries were more often affected by radiation therapy (compared with the circumflex artery). In our study, mean LAD maximum dose was 39.5 Gy for 3DCRT and 29.17 Gy for IMRT ( $\mathrm{p}=0.03)$.

\section{Lung dose}

The occurrence of radiation pneumonitis (RP) is related to the ipsilateral lung volume irradiated.22 In our study, the ipsilateral lung V20Gy for IMRT (22.4\%) is significantly less than that for 3DCRT $(37.9 \%$; $<0.01)$. Ipsilateral lung mean dose was also higher in 3DCRT (20.29 Gy) compared to the one in IMRT $(16.51 \mathrm{~Gy})(\mathrm{p}<0.01)$. Both the lung V20Gy and 
mean dose were significantly lower in IMRT than in 3DCRT ( $\mathrm{p}<0.01$ ). Contra lateral lung $\mathrm{V}_{5 \mathrm{~Gy}}$ and mean dose of both the plans showed no significant differences. The mean lung doses (MLD) of both lung were higher compared to the report from Marks et $a l^{23}$, and this may be due to larger breast volumes in our study. Since there is no absolute safe MLD below which there is no pneumonitis, the clinically acceptable risk of RP depends on the risk-benefit ratio of the individual patient selection basis.

\section{Secondary malignancy}

The IMRT plans contributed a modestly higher dose to adjacent healthy soft tissues. In our study, the mean V5Gy volume for 3DCRT was much lower than that of IMRT. The main concern with healthy tissue dose increases of this magnitude is an increased risk of late second malignancy. ${ }^{24,25}$ Some investigators suggest that IMRT might increase the incidence of secondary cancer from $1 \%$ in conventional planning to $1.75 \%$ in IMRT planning for patient's surviving 10 years. $^{24}$

Furthermore, the treatment Monitor Unit (MU) was significantly higher in IMRT technique. The monitor unit for IMRT is 6-8 times more than 3DCRT is a concern. ${ }^{24,26,27}$ This in turn shows that the integral dose would be higher. Pirzkall et al. ${ }^{28}$ studied that the integral dose for IMRT was higher than conventional treatment. Similar observation was made in our study as integral dose for IMRT was $22 \%$ higher than that for 3DCRT. This higher integral dose was probably due to increased number of beams used in IMRT than in 3D CRT, thus involving larger volume of healthy tissue during IMRT plan optimization. Modulation of beams also increases the treatment time during treatment delivery. Furthermore, the leakage and scatter dose to non-target tissue of the patients will be proportional to the number of monitor units used. Few studies ${ }^{13,} 29$ have found to have increased low dose volumes with increasing beam angles.

High integral dose attributed to second malignancy, which is likely to be of greatest concern in younger women and in patients with a low risk for systemic relapse that are likely to live for many years after the diagnosis of breast cancer. ${ }^{27}$ There have been reports ${ }^{24}$ suggesting that adjuvant radiation therapy for breast cancer may increase the risk of lung cancer and angiosarcoma. The risk of sarcoma in the treated volume is likely to be similar with IMRT or standard techniques, but it is possible that second primary lung cancers might be increased by IMRT, especially if the woman is a smoker. ${ }^{27}$ Therefore, individual assessment of treatment volume goals and longevity of patients with and without radiation therapy is necessary in order to balance the short to medium-term benefits of reducing the volume of critical structures, especially heart and lung, receiving higher radiation dose.

\section{Respiratory gating}

Organ motion during the IMRT treatment has been accounted for using real-time position management (RPM;
Varian Medical System, Palo Alto, California, USA). The RPM system supports automatic on and off triggering of radiation beam during the treatment. The marker position approximates identical and in-phase alignment of breast and marker motion. Due to breathing motion, the PTV may move outside the external contour as defined on the planning CT and result in a geographic miss of the target. Although the geometric uncertainties and intra fraction movement are taken into account on PTV margin, but the breast is a superficial organ and often the CTV will extend to the skin surface. In these cases, the restriction of the PTV to 3 $\mathrm{mm}$ from the skin surface will not provide an adequate margin for intra-fraction breathing motion..$^{29-33}$ The main concern would be the CTV being under-dosed. In order to use gating, the PTV motion must be in phase with the breathing cycle or must at least be able to be predicted from the breathing cycle using technology such as RPM. Conformal blocking and breath-hold techniques can essentially eliminate the heart from the primary beams. Historically, whole heart doses up to $30 \mathrm{~Gy}$ were reasonably well tolerated. ${ }^{34-36}$

\section{Conclusion}

IMRT for breast cancer treatment is feasible. In comparison to 3DCRT, IMRT reduced the maximum dose to the target volume, and dose to OAR was reduced too. However, 3DCRT technique was superior in terms of low dose volume of normal tissue, integral dose, and treatment time. Consequences of these low doses would have to be weighed against the benefits of reducing high doses on individual patient selection basis. With the use of breath-hold gated technique in IMRT, it can further improve the target coverage and reduction of doses to the heart, lung, and LAD. SIB technique could reduce the overall treatment duration by about one week.

\section{Competing interests}

The authors declare that they have no conflicts of interest. The authors alone are responsible for the content and writing of the paper.

\section{References}

1. Overgaard M, Nielsen HM, Overgaard J. Is the benefit of post mastectomy irradiation limited to patients with four or more positive nodes, as recommended in international consensus reports? A subgroup analysis of the DBCG 82 b \& c randomized trials. Radiother Oncol 2007; 82:247-253.

2. Hong L, Hunt M, Chui C, Spirou S, Forster K, Lee H, Yahalom J, Kutcher GJ, McCormick B. Intensity modulated tangential beam irradiation of the intact breast. Int J Radiat Oncol Biol Phys 1999; 44:1155-64. 
3. Prescribing, recording and reporting photon beam therapy. International commission on Radiological Units and Measurements (ICRU) Report \# 50: ICRU1993.

4. Prescribing, recording and reporting photon beam therapy. International commission on Radiological Units and Measurements (ICRU) Report \# 62: ICRU 1997.

5. Baltas D, Kolotas C, Geramani K, Mould RF, Ioannidis G, Kekchidi M, Zamboglou N. A conformal index (COIN) to evaluate implant quality and dose specification in brachytherapy. Int J Radiat Oncol Biol Phys 1998; 40: 515-24.

6. Knoos T, Kristensen I, Nilsson P. Volumetric and dosimetric evaluation of radiation treatment plans: Radiation Conformity Index. Int J Radiat Oncol Biol Phys 1998; 42: 1169-76.

7. Paddick I. A simple scoring ratio to index the conformity of radiosurgical treatment plans. Technical note. JNeurosurg 2000; 93:219-22.

8. Prescribing, recording and reporting photon beam therapy. International commission on Radiological Units and Measurements (ICRU) Report \# 83: ICRU 2010.

9. Mohan R, Wu Q, Manning M, Schmidt-Ullrich R. Radiobiological considerations in the design of fractionation strategies for intensity-modulated radiation therapy of head and neck cancers. Int $J$ Radiat Oncol Biol Phys 2000; 46:619-630.

10. Zhou GX, Xu SP, Dai XK, et al. Clinical dosimetric study of three radiotherapy techniques for postoperative breast cancer: Helical Tomotherapy, IMRT, and 3D-CRT. Technol Cancer Res Treat 2011;10:15-23.

11. Selvaraj RN, Beriwal S, Pourarian RJ, et al. Clinical implementation of tangential field intensity modulated radiation therapy (IMRT) using sliding window technique and dosimetric comparison with 3D conformal therapy (3DCRT) in breast cancer. Med Dosim 2007; 32:299-304.

12. Moody AM, Mayles WP, Bliss JM, et al. The influence of breast size on late radiation effects and association with radiotherapy dose inhomogeneity. Radiother Oncol 1994; 3:106-112.

13. Chui CS, Hong L, Hunt M, McCormick B. A simplified intensity modulated radiation therapy technique for the breast. Med Phys 2002; 29:522-529.

14. Donovan EM, Bleackley NJ, Evans PM, Reise SF, Yarnold JR. Dose-position and dose-volume histogram analysis of standard wedged and intensity modulated treatments in breast radiotherapy. $\mathrm{Br} J$ Radiol 2002; 75:967-973.

15. Stewart JR, Fajardo LF, Gillette SM, Constine LS. Radiation injury to the heart. Int I Radiat Oncol Biol Phys 1995; 31:1205-12.
16. Gagliardi G, Constine LS, Moiseenko V, et al. Radiation dose-volume effects in the heart. Int J Radiat Oncol Biol Phys 2010; 76:S77-85.

17. Rongsriyam K, Rojpornpradit P, Lertbutsayanukul $\mathrm{C}$, et al. Dosimetric study of inverse-planed intensity modulated, forward-planned intensity modulated and conventional tangential techniques in breast conserving radiotherapy. J Med Assoc Thai 2008; 91:1571-1582.

18. Ragaz J, Olivotto IA, Spinelli JJ, et al. Locoregional radiation therapy in patients with high-risk breast cancer receiving adjuvant chemotherapy: 20-Year results of the British Columbia Randomized trial. $J$ Natl Cancer Inst 2005; 97:116-126.

19. Høst H, Brennhovd I, Loeb M. Postoperative radiotherapy in breast cancer - long term results from the Oslo study. Int J Radiat Oncol Biol.Phys 1986;12:727-732.

20. Giordano SH, Kuo Y, Freeman JL, et al. Risk of cardiac death after adjuvant radiotherapy for breast cancer. J Natl Cancer Inst 2005; 97:419-424.

21. Boivin JF, Hutchison GB, Lubin JH, et al. Coronary artery disease mortality in patients treated for Hodgkin's disease. Cancer 1992; 69:1241-1247.

22. Recht A, Ancukiewicz M, Alm El-Din MA, et al. Lung dose-volume parameters and the risk of pneumonitis for patients treated with accelerated partial-breast irradiation using three-dimensional conformal radiotherapy. I Clin Oncol 2009; 27:3887-3893.

23. Marks LB, Bentzen SM, Deasy JO, et al. Radiation dose-volume effects in the lung. Int J Radiat Oncol Biol Phys 2010; 76:S70-76.

24. Hall EJ, Wuu CS. Radiation-induced second cancers: The impact of 3D-CRT and IMRT. Int J Radiat Oncol Biol Phys 2003; 56:83-88.

25. Gao X, Fisher SG, Emami B. Risk of second primary cancer in the contralateral breast in women treated for early-stage breastcancer: A population-based study. Int $J$ Radiat Oncol Biol Phys 2003; 56:1038-1045.

26. Jothybasu KS, Bahl A, Subramani V, et al. Static versus dynamic intensity-modulated radiotherapy: Profile of integral dose in carcinoma of the nasopharynx. JMed Phys 2009; 34:66-72.

27. Followill D, Geis P, Boyer A. Estimates of whole-body dose equivalent produced by beam intensity modulated conformal therapy. Int J Radiat Oncol Biol Phys 1997; 38:667-672.

28. Pirzkall A, Carol M, Lohr F, et al. Comparison of intensity-modulated radiotherapy with conventional conformal radiotherapy for complex-shaped tumors. Int J Radiat Oncol Biol Phys 2000; 48:1371-1380.

29. Cho BCJ, Schwarz M, Mijnheer BJ, Bartelink H. Simplified intensity-modulated radiotherapy using pre-defined segments to reduce cardiac complica- 
tions in left-sided breast cancer. Radiother Oncol 2004; 70:231-41.

30. Pedersen AN, Korreman S, Nystrom $\mathrm{H}$, et al. Breathing adapted radiotherapy of breast cancer: reduction of cardiac and pulmonary doses using voluntary inspiration breath-hold. Radiother Oncol 2004; 72:53-60.

31. Kron T, Perera F, Lee C, Yu E. Evaluation of intraand inter fraction motion in breast radiotherapy using electronic portal cine imaging. Technol Cancer Res Treat 2004; 3:443-50.

32. Wong JW, Sharpe MB, Jaffray DA, et al. The use of active breathing control (ABC) to reduce margin for breathing motion. Int J Radiat Oncol Biol Phys 1999; 1:911-9.

33. Jagsi R, Moran JM, Kessler ML, et al. Respiratory motion of the heart and positional reproducibility under active breathing control. Int I Radiat Oncol Biol Phys 2007; 68:253-258.
34. Gagliardi G, Constine LS, Moiseenko V, Correa C, Pierce LJ, et al. Radiation dose-volume effects in the heart. Int J Radiat Oncol Biol Phys 2010; 76: S77-85.

35. Giraud P, Djadi-Prat J, Morvan E, Morelle M, Remmonay R, Pourel N, Durdux C, Carrie C, Mornex F, Le Péchoux C, Bachaud JM, Boisselier P, Beckendorf V, Dendale R, Daveau C, Garcia R; les centres investigateurs du Stic. Dosimetric and clinical benefits of respiratory-gated radiotherapy for lung and breast cancers: results of the STIC 2003. Cancer Radiother 2012; 16:272-81.

36. Stephen C, James R, Derek W, Lee S, Ivo AO. Respiratory Gating: Using Deep Inspiration Breath Hold Radiation Therapy to Treat Left Breast Cancer. Journal of Medical Imaging and Radiation Sciences 2008; 39:192-197. 